\title{
Aplicação de método de coloração tricrômica, em fezes diarréicas de infectados pelo HIV, para pesquisa de microsporídios
}

\author{
Trichrome staining method applied in stools from HIV-infected patients with \\ diarrhoea, for microsporidia investigation
}

\author{
Juliane Gomes de Paula Amato, Valdir Sabbaga Amato e Vicente Amato Neto
}

\begin{abstract}
Resumo Os microsporídios são implicados em várias manifestações clínicas em pacientes com AIDS; os quadros diarréicos são os mais comuns. O diagnóstico das microsporidioses dependia da microscopia eletrônica para exame de materiais obtidos por procedimentos invasivos. A técnica de coloração tricrômica modificada permite o diagnóstico sem necessidade deste procedimento, através da microscopia óptica. No presente estudo foi aplicado o método de coloração tricrômica em fezes de 62 pacientes com diarréia, infectados pelo HIV ou com AIDS. Das 62 amostras, identificou-se esporos de microsporídios em uma. 0 trabalho corrobora a presença destes protozoários em nosso meio, associada a quadros de diarréia crônica em pacientes com AIDS e grave comprometimento imunológico; constata que este método de coloração promove satisfatória visualização de esporos de microsporídios em fezes e aponta caminhos para novos estudos.
\end{abstract}

Palavras-chaves: Microsporídios. Coloração tricrômica modificada. AIDS.

\begin{abstract}
The microsporidia have been involved in several clinical manifestations in patients with AIDS, of whom diarrhoea is the commonest. The diagnosis of microsporidiasis depended on invasive procedures and the identification of the organisms is made by electron microscopy. The modified trichrome staining method allows that the diagnosis be made without such procedures by using light microscopy. In the present work, the modified trchrome method was applied in stools from 62 patients with diarrhoea, who had asymptomatic HIV infection or AIDS. Of the 62 samples analyzed, there was detection of microsporidial spores in one. This work confirms the existence of such protozoans in our patients, associated with manifestations of chronic diarrhoea in patients with AIDS who have severe immunodeficiency and ascertains that this staining method allows satisfactory identification of microsporidia from faeces, as well points out some directions to further studies.
\end{abstract}

Key-words: Microsporidia. Modified trichrome staining. AIDS

As microsporidioses são infecções causadas por protozoários intracelulares obrigatórios, ubiqüitários, pertencentes ao filo Microspora, os quais são genericamente designados pelo termo não taxonômico de microsporídios 3491619.
Antes da pandemia da AIDS, as infecções por estes protozoários eram extremamente raras, havendo na literatura apenas dez casos, suficientemente bem documentados, de microsporidiose não associada ao vírus da

\footnotetext{
Departamento de Doenças Infecciosas e Parasitárias do Hospital das Clínicas da Faculdade de Medicina da Universidade de São Paulo, São Paulo, SPe Laboratório de Investigação Médica - Parasitologia do Hospital das Clínicas, da Faculdade de Medicina da Universidade de São Paulo, SP, Brasil.

Endereço para correspondência: Dr. a Juliane Gomes de Paula Amato. Alameda Gabriel Monteiro da Silva 429, 01441-000 São Paulo, $\mathrm{SP}$, Brasil.

Fax: 5511 881-8158.

Recebido para publicação em 17/4/98.
} 
imunodeficiência humana1 610152122233033 . Por outro lado, desde 1985 quando o primeiro caso de microsporidiose em paciente com AIDS foi descrito na França por Modigliane et al24, um crescente número de casos relacionados a esta condição de comprometimento imunológico vem sendo descrito em várias regiões do mundo, envolvendo um espectro cada vez mais abrangente de manifestações clínicas, sendo os quadros diarréicos os mais comuns.

O método de coloração tricrômica modificado por Weber et al38 constitui significativo avanço, pois possibilita a identificação dos esporos de microsporídios em materiais de fácil obtenção, como fezes e urina, através do emprego da microscopia óptica, viabilizando assim, a realização do diagnóstico em um maior número de pacientes sem necessidade de procedimentos invasivos 2632353842 . O presente estudo tem por objetivo desenvolver experiência com a aplicação deste método diagnóstico, o qual é perfeitamente viável e facilmente aplicável aos laboratórios de Parasitologia, e iniciar um melhor entendimento sobre estas infecções em nosso meio.

\section{MATERIAL E MÉTODOS}

Entre maio de 1995 e janeiro de 1998, foram arrolados 60 indivíduos adultos com infecção pelo HIV diagnosticada pelo método de ELISAe confirmada pela técnica de Western blot, os quais apresentavam ou acometimento assintomático pelo HIV ou AIDS, de acordo com os critérios estabelecidos pelos "Centers for Diseases Control and Prevention" (CDC)12, além de duas crianças filhas de mães com AIDS. Para inclusão no estudo deveria haver diarréia, definida como três ou mais episódios de fezes líquidas ou pastosas no período de 24 horas 38 . De cada indivíduo foi colhida uma amostra de material fecal disposta em dois frascos, um contendo formalina a $10 \%$ e outro sem conservante. As amostras foram analisadas no Laboratório de Investigação Médica-Parasitologia do HC-FMUSP, onde foram submetidas à pesquisa de protozoários e helmintos através dos métodos de Hoffmann et al18, Faust et al17 e Kinyoun20. A pesquisa de esporos de microsporídios foi efetuada segundo o método da coloração tricrômica modificado, desenvolvido por Weber et al38. Sempre que possível, foi obtida a contagem de linfócitos CD4, efetuada até no máximo um mês antes ou após a coleta do material fecal.

\section{RESULTADOS}

As características demográficas dos 62 indivíduos estão resumidas na Tabela 1. Destes, 16 apresentavam infecção assintomática pelo HIV $(25,8 \%)$ e 46 tinham AIDS (74,2\%). A média da contagem de linfócitos $\mathrm{CD}_{4}$ dos 60 que dispunham deste exame foi de 157,2 células $/ \mathrm{mm}^{3}$. Os pacientes em sua maioria apresentavam diarréia líquida (79\%), e o restante, fezes pastosas $(21 \%)$. O tempo de duração da diarréia foi menor que sete dias em $12(19,4 \%)$, de sete dias a um mês em 21 (33,9\%), e acima de um mês em 29 $(46,7 \%)$. Existiram sintomas associados ao quadro diarréico em $64,5 \%$, os quais estão especificados na Figura 1.

Dentre as 62 amostras de fezes, houve identificação de esporos de microsporídios em apenas uma. Estes esporos apresentavam as mesmas características relatadas por Weber et al 38, ou seja, estruturas ovóides de aproximadamente $1,5 \times 0,9 \mu \mathrm{m}$, coradas em

Tabela 1 - Dados demográficos dos pacientes com AIDS e diarréia incluídos no estudo $(n=62)$.

\begin{tabular}{llrr} 
& média \pm dp & variação & \multicolumn{2}{c}{$35,5 \pm 10,5$} \\
& & $\mathrm{n}^{\circ}$ & $(\%)$ \\
\hline Sexo & & 54 & 87,1 \\
& masculino & 8 & 12,1 \\
Raça & feminino & 38 & 61,3 \\
& branca & 4 & 6,5 \\
& negra & 19 & 30,6 \\
& parda & 1 & 1,6 \\
\hline
\end{tabular}

$\mathrm{dp}=$ desvio padrão. 


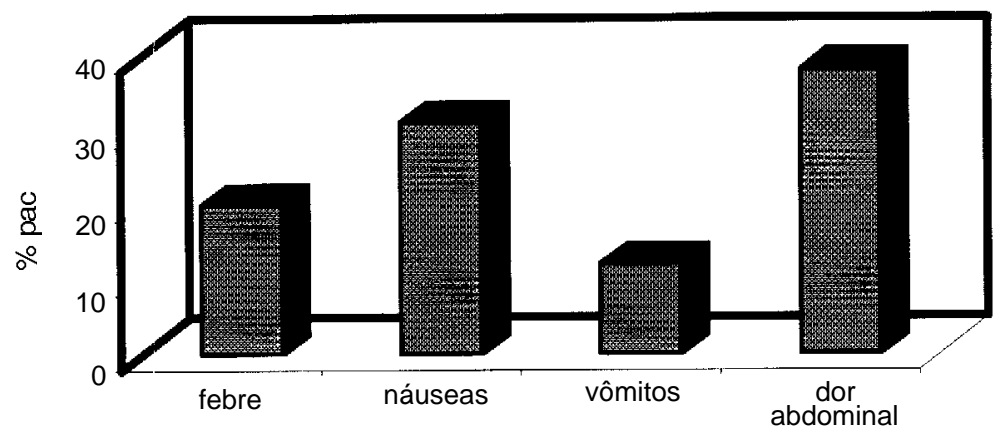

Figura 1 - Porcentual de pacientes com sintomas/sinais associados à diarréia, nos 62 pacientes incluídos no estudo.

vermelho rosado brilhante, com distinta faixa disposta equatorial ou diagonalmente, deixando assim evidente uma extremidade

descorada (Figura 2). Outros achados do exame parasitológico estão expostos na Tabela 2.

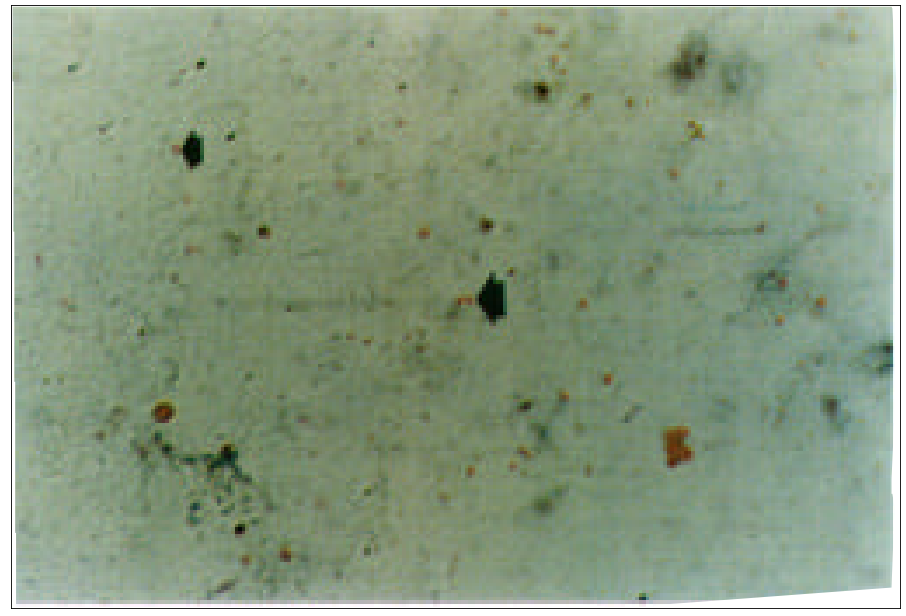

Figura 2 - Esfregaço de amostra de fezes fixada em formalina, com evidência de esporos de microsporídios corados em vermelho rosado; alguns esporos mostram faixa rosada (setas). Bactérias estão coradas em verde esmaecido (aumento de 1.000x).

Tabela 2 - Resultados do exame parasitológico, de fezes em 62 pacientes com AIDS e diarréia.

\begin{tabular}{lcc}
\hline & $\mathrm{n}-$ & $\%$ \\
\hline Isospora sp & 5 & 8,1 \\
Cryptosporidium sp & 4 & 6,5 \\
Giardia lamblia & 3 & 4,8 \\
Endolimax nana & 1 & 1,6 \\
Ancilostomídeo & 1 & 1,6 \\
Microsporídeo & 1 & 1,6 \\
lodamoeba bütschlii & 1 & 1,6 \\
nenhum & 46 & 74,2 \\
\hline
\end{tabular}




\section{DISCUSSÃO}

O método de coloração tricrômica modificado por Weber et al38 representa um verdadeiro progresso para o diagnóstico das microsporidioses humanas, pois provê uma fácil detecção dos esporos em materiais de simples obtenção, como fezes e urina, sem ter que submeter o paciente a procedimentos invasivos, sendo considerado o método de coloração padrão para o diagnóstico destas parasitoses através da microscopia óptica26 32353842 . A evidência de esporos de microsporídios em um dos 62 pacientes da presente amostra, constata a existência destes protozoários em nosso meio. O paciente em questão, era do sexo masculino, tinha 31 anos, diagnóstico de AIDS e apresentava diarréia por tempo superior a um mês, com fezes de consistência pastosa, sintomas associados de náuseas e dor abdominal, possuindo uma contagem de linfócitos $C_{4}$ de 35 células $/ \mathrm{mm}^{3}$. Estas características estão de acordo com as referidas na literatura, a qual relata, na maioria das vezes, casos de microsporidiose intestinal em indivíduos com AIDS, gravemente imunodeficientes, apresentando contagem de linfócitos $\mathrm{CD}_{4}$ inferiores a 100 células $/ \mathrm{mm}^{3}$, com diarréia de longa duração2 11252728293436373839404243 .

Entretanto, igualmente é relatado na literatura a presença de microsporídios associada a quadros de diarréia em pessoas com infecção pelo HIV e menor grau de comprometimento imunológico2 134142 , pacientes com AIDS e diarréia de evolução autolimitada ${ }^{43}$, assim como em imunocompetente não infectado pelo HIV, com diarréia auto-limitada e apresentando contagem de linfócitos $C D_{4}$ de 2 a 052 células $/ \mathrm{mm}^{3}$ 33; também é relatado paciente submetido a transplante de coração e pulmão, não infectado pelo HIV, com diarréia crônica e contagem de linfócitos $\mathrm{CD}_{4}$ superior a 300 células $/ \mathrm{mm}^{3} 30$, além de haver publicação de um caso de diarréia em criança infectada pelo HIV40. Estes relatos serviram de base para a decisão de incluir no presente estudo pacientes com diarréia de mais curta duração, tivessem eles AIDS ou infecção assintomática, e também crianças, não somente indivíduos com AIDS que sofresses de diarréia crônica e grave deficiência imunológica.

Todavia, o encontro de esporos de microsporídios em apenas $1,6 \%$ da amostra poderia estar relacionado a pelo menos três fatores. O primeiro, referente à observação referida por alguns autores de que a excreção de esporos é aparentemente feita de maneira irregular à semelhança de outros parasitas intestinais e, sendo assim, poderia não ser detectada em uma única amostra de material fecal5 9 13. O outro diz respeito à relação, constatada recentemente por Clarridge et al13, entre a abundância de parasitas em material de biópsia intestinal, aliada à presença de esporos em amostras de fezes, sendo pois, a detecção de esporos em material fecal tanto mais fácil quanto maior a quantidade de esporos e estágios plasmodiais presente nos tecidos. Estes autores constataram esporos de microsporídios ao exame de espécimes fecais em $100 \%$ dos pacientes com moderada ou abundante quantidade de parasitas nos tecidos, ao passo que este número decrescia para $57 \%$ nos pacientes com pequena quantidade tecidual de parasitas; conseqüentemente, mesmo em um paciente infectado por microsporídios, a pesquisa de esporos nas fezes pode resultar negativa se o grau de parasitismo da mucosa intestinal for pequeno. O terceiro fator pode estar correlacionado ao tamanho da amostra estudada. Embora os casos começaram a ser incluídos em maio de 1995, eles foram mais efetivamente coletados de janeiro de 1996 a janeiro de 1998, período este que veio coincidir com importantes avanços na terapêutica para AIDS. Com o advento das associações de drogas antiretrovirais, observou-se uma sensível redução nos casos de infecções oportunistas, especialmente com relação aos casos de diarréia. Evidentemente este decréscimo prejudicou a ampliação da presente amostra.

Seria pertinente ressaltar que a maioria dos estudos que relatam casos de microsporidiose intestinal envolve populações altamente selecionadas, ou seja, pacientes com AIDS e pronunciada imunodeficiência, com diarréia crônica sem etiologia definida, a despeito da extensa e minuciosa investigação diagnóstic a, ou grave síndrome mábsortiva2 7814242527282931394144 . Portanto, as prevalências extraídas destes trabalhos, que variam de 2 a $50 \%$, não refletem a real freqüência da associação das microsporidioses com acometimentos intestinais, a qual, pois, para ser estabelecida dependerá de estudos com populações maiores e mais abrangentes.

Os outros achados ao exame parasitológico expressam agentes comuns envolvidos na etiologia de distúrbios intestinais na população 
em geral, como Giardia lamblia e ancilostomídeo, e propriamente nos pacientes com AIDS como, Isospora $s p$ e Cryptosporidium $s p$, enquanto que protozoários considerados não patogênicos também foram identificados, como Endolimax nana e lodamoeba bütschilii. Não foi objetivo deste trabalho estudar os agentes etiológicos das diarréias de pacientes com AIDS ou infecção assintomática pelo HIV, sendo a pesquisa de cistos, ovos e larvas apenas uma complementação do exame de fezes, e que serviu ao intuito de melhor assistir os pacientes do ponto de vista da investigação diagnóstica.

Muitas lacunas referentes ao estudo das microsporidioses humanas permanecem sem esclarecimento, especialmente em nosso meio. Dúvidas sobre se sua patogenicidade ocorre somente frente a situações de comprometimento imunológico ou se poderia ser responsável por muitos quadros autolimitados em indivíduos imunocompetentes; questões a respeito da existência de portadores sãos, bem como distribuição geográfica, reservatórios, modos de transmissão, possíveis tratamentos e controle de cura. Estes grandes hiatos somente começaram a ser preenchidos mediante o aumento da experiência por parte dos laboratórios com técnicas diagnósticas aplicáveis não somente aos grandes centros de pesquisa, mas igualmente aos laboratórios de rotina, com finalidade assistencial, como é o caso do método de coloração tricrômica modificado por Weber et al38. Entretanto, paralelamente será mais prudente proceder a um amplo estudo comparativo entre as diversas técnicas de coloração para microsporídios, que se vêm proliferando na literatura em anos recentes, inclusive contrapondoas ao exame histopatológico pela microscopia óptica e eletrônica, para estabelecer a verdadeira sensibilidade e especificidade de cada uma. Todos os demais aspectos dos estudos das microsporidioses, como imunofisiopatogenia e terapêutica, estarão estritamente vinculados a estes esclarecimentos fundamentais.

\section{REFERÊNCIAS BIBLIOGRÁFICAS}

1. Asmuth DM, De Girolami PC, Federman M, Ezratty CR, Pleskow DK, Desai G, Wanke CA. Clinical features of microsporidiosis in patients with AIDS. Clinical Infectious. Diseases 18:819-825, 1994

2. Ashton N, Wirasinha PA. Encephalitozoonosis (Nosematosis) of the cornea. British Journal of Ophthalmology 57:669-674, 1973.

3. Atías AM. Actualizaciones sobre microsporidiosis en el hombre. Revista Médica de Chile 123:762-772, 1995.

4. Beauvais $B$, Sarfati $C$, Larivière $M$, Derouin $F$. Microsporidioses. La Presse Médicale 23:332-338, 1994.

5. Beauvais B, Sarfati C, Molina JM, Lesourd A, Larivière M. Comparative evaluation of five diagnostic methods for demonstrating microsporidia in stool and biopsy specimens. Annals of Tropical Medicine and Parasitology 87:99-102, 1993.

6. Bergquist NR, Stintzing G, Smedman L, Waller T, Anderson T. Diagnosis of encephalitozzonosis in man by serological tests. British Medical Journal 288:902, 1984.

7. Bouchaud O, Houzé S, Saada M, Lebras J, Coulaud JP. Intestinal microsporidiosis in AIDS patients: high percentage of asymptomatic carriers. In: Abstract of the International Conference on AIDS, Berlin, v1, p. 387, 1993.

8. Bretagne S, Foulet F, Alkassoum W, Fleury-Feith J, Develoux MP. Prevalence des spores d' Enterocytozzon bieneusi dans les selles de patients sidéens et d'enfants africains non infectes par le VIH. Bulletin de La Sociéte de Pathologie Exotique 86:351-357, 1993.
9. Bryan RT. Microsporidia. In: Mandel GL, Bennet JE, Dolan R (eds) Principles and pratice of infectous diseases. 4th edition. New York, Churchill Livingstone, p.2513-2524, 1997.

10. Cali A, Meisler DM, Lowder CY, Lembach R, Ayers L, Takvorian PM, Rutherford I, Longworth DL, McMahon J, Bryan RT. Corneal microsporidioses: characterization and identification Journal of Protozoology 38:215S217S, 1991.

11. Caramello P, Mazzucco G, Romeo M, Ullio A, De Rosa G, Lucchini A, Forno B, Brancale T, Macor A, Preziosi C, Gioannini P. Clinical and microscopical features of smallintestinal microsporidiosis in patients with AIDS. Infection 23:362-368, 1995.

12. Centers for diseases control and prevention: revised classification system for HIV infection and expanded surveillance case definition for AIDS among adolescents and adults. MMWR 41 [RR-17]:1-19, 1992.

13. Clarridge III JE, Karkhanis S, Rabeneck L, Marino B, Foote LW. Qujantitative light microscopic detection of Enterocytozoon bieneusi in stool specimens: a longitudinal study of human immunodeficiency virus-infected microsporidiosis paritnts. Journal of Clinical Microbiology 34:520-523, 1996.

14. Cotte L, Rabodonirina M, Piens MA, Perreard M, Mojon $\mathrm{M}$, Trepo C. Prevalence of intestinal protozoans in French patients infected with HIV. Journal of Acquired Immunel Deficiency Syndromes 6:1024-1029, 1993.

15. Davis RM, Font RL, Keisler MS, Shadduck JÁ. Corneal microsporidiosis. A case report including ultrastrutural observations. Ophthalmology 97:953-957, 1990. 
16. Deluol AM, Cenac J. Les microsporidioses Microsporidiosis. Les Annales du Biologique et Clinique (Paris) 52:37-44, 1994.

17. Faust EC, D'Antoni JS, Odom V. Miller MJ, Peres C, Sawitz W, Thomen LF, Tobie J, Walker JH. A critical study of clinical laboratory technics for the diagnosis of protozoan cystis and helminth eggs in faeces preliminary communication. American Journal of Tropical Medicine 18:169-183, 1938.

18. Hoffman WA, Pons JA, Janer JL. The sedimentationconcentration method in schistosomiasis mansoni. Puerto Rico Journal of Public Health 9:281-298, 1934.

19. Levine ND, Corliss JO, Cox FEG, Deroux JG, Grain J, Honigberg BM, Leedale GF, Loeblich AR; Lom J, Lynn D, Merinfield EG, Page FC, Poljansky G, Sprague V, Vavra J, Wallace FG. A newly revised classification of the protozoa. Journal of Protozoology 27:37-58, 1980.

20. Ma P, Soave R. Three-setp stool examination for cryptosporidiosis in 10 homosexual men with protacted watery diarrhoea. Journal of Infectious Diseases 147:824-828, 1988.

21. Macher AM, Neafie R, Agritt P, Tuur SM. Microsporidial myositis and the acquired immunodeficiency syndrome (AIDS): a four-year follow-up. [Letter]. Annals of Internal Medicine 109:343, 1988.

22. Margileth AM, Strano AJ, Chandra R, Neafie R, Blum M, McCully LCRM. Disseminated nosematosis in na immunologically compromised infant. Archives of Pathology 95:145-150, 1973.

23. Matsubayashi $\mathrm{H}$, Koike $\mathrm{T}$, Mikata $\mathrm{T}$, Hagiwara $\mathrm{S}$. A case of Encephalitozoon-like body infection in man. Archives of Pathology 67:181-187, 1959.

24. Modigliani R, Bories $C$, Le Charpentier $Y$, Salmeron M, Messing B, Gallian A, Rambaud JC, Labergne A, Cochand-Priollet B, Desportes I. Diarrhoea and malabsorption in acquired immunodeficiency syndrome: a study of four cases with special emphasis on oportunistic protozoan infestations. Gut 26:179-187, 1985.

25. Molina JM, Sarfati C, Beauvais B, Lémann M, Lesourd A, Ferchal F, Casin I, Lagrange F, Modigliani R, Derouin F. Intestinal microsporidiosis in human immunodeficiency virus-infected patients with chronic unexplained diarrhoea: prevalence and clinical and biologic features. Journal of Infectious Diseases 167:217-221, 1993.

26. Moura H, Da Silva JLN, Sodré FC, Brasil P, Wallmo K, Wahlquist S, Wallace S, Croppo GP, Visvesvara GS. Gram-chromotrope: a new technique that enhances detection of microsporidial spores in clinical samples. Journal of Eukaryotic Microbiology 43:94S-95S, 1996.

27. Orestein JM. Microsporidiosis in the acquired immunodeficiency syndrome. Journal of Parasitology 77:843-864, 1991.

28. Orestein JM, Chiang J, Steinberg W, Smith PD, Rotterdam H, Kotler DP. Intestinal microsporidiosis as a case of diarrhoea in human immunodeficiency virusinfected patients: a report of 20 cases. Human Pathology 21:475-481, 1990.

29. Pitisuttithum $P$, Chindanond D, Phibbonnakit D, Leelasuphasri S. Microsporidium: modified technique for light microscopic diagnosis. Journal of Medicine Associated Thailand 78:251-254, 1995.

30. Rabeneck L, Genta RM, Gyorkey F, Clarridge JE, Gyorkey P, Foote LW. Observations on the pathological spectrum and clinical course of microsporidiosis in men infected with the human immunodeficiency virus: followup study. Clinical of Infectious Diseases 20:1229-1235, 1995.

31. Rijpstra AC, Canning EU, Van Ketel RJ, Schattenkerk JKME, Laarman JJ. Use of light microscopy to diagnose small-intestinal microsporidiosis in patient with AIDS. Journal of Infectious Diseases 157:827-830, 1988.

32. Rosberger DF, Serdarevic ON, Erlandson RA. Successful treatment of microsporidial keratoconjunctivitis with topical fumagillin in a patient with AIDS. Cornea 12:261265, 1993.

33. Sandfort J, Hannemann A, Gelderblom H, Schneider T, Ruf B. Intestinal microsporidiosis in patients with HIVinfection. In: Abstracts of the International Conference on AIDS, Berlin, v1, p. 383, 1993.

34. Schattenkerk JKME, Karel J, Van Gool T, Bartelsman JFWM, Lange JMA, Reiss P. Metronidazole for microsporidium-associated diarrhoea in symptomatic HIV-1 infection. In: Abstracts of the International Conference on AIDS, Florence, v2, p. 248, 1991.

35. Schwartz DA, Visvesvara GS, Leitch GJ, Tashljian L, Pollack M, Holden J, Bryan RT. Pathology of symptomatic microsporidial (Encephalitozoon hellem) bronchiolitis in the acquired immunodeficiency syndrome: a new respiratory pathogen diagnosed from lung biopsy bronchoalveolar lavage, sputum, and tissue culture. Human Pathology 24:937-943, 1993.

36. Van Gool T, Hollister WS, Schattenkerk JKME, Van Den Bergh Weerman MA, Terpstra WJ, Van Ketel, RJ, Reiss $\mathrm{P}$, Canning EU. Diagnosis of Enterocytozoon bieneusi microsporidiosis in AIDS patients by recovery spores from faeces. Lancet 336:697-698, 1990.

37. Wanke CA, Mattia AR. A 36-year-old man with AIDS, increase in chronic diarrhoea and intermittent fever and chills. New England Journal of Medicine 329:1946-1954, 1993.

38. Weber R, Bryan RT. Microsporidial infections in immunodeficient and immunocompetent patients. Clinical Infectious Diseases 19:517-521, 1994.

39. Weber R, Kuster H, Keller R, Bachi T, Spycher MA, Briner J, Russi E. Lüthy R. Pulmonary and intestinal microsporidiosis in a patient with acquired immunodeficiency syndrome. American Review of Respiratory Diseases 146:1603-1605, 1992. 
Revista da Sociedade Brasileira de Medicina Tropical 32:277-283, mai-jun, 1999.

40. Weber R, Kuster H, Visvesvara GS, Bryan RT, Schwartz $\mathrm{Da}$, Lüthy $\mathrm{R}$. Disseminated microsporidiosis due to Encephalitozoon hellem: pulmonary colonization, microhematuria, and mild conjunctivitis in a patient with AIDS. Clinical Infectious Diseases 17:415-419, 1993.

41. Weber R, Müller A, Spycher MA, Opravil M, Ammann R, Briner J. Intestinal Enterocytozoon bieneusi microsporidiosis in an HIV-infected patient: diagnosis by ileo-colonoscopic biopsies and long-term follow-up. Clinical Investigation 70:1019-1023, 1992.

42. Weber R, Sauer B, Luthy R. Intestinal coinfection with Enterocytozoon bieneusi and Cryptosporidium in a human immunodeficiency virus-infected child with chronic diarrhoea. Clinical Infectious Diseases 17:480-483, 1993.

43. Wittner M, Tanowitz HB, Weiss LM. Parasitic infections in AIDS patients: cryptosporidiosis, isosporiasis, microsporidiosis, cyclosporiasis. Infectious Diseases Clinical of North America 7:569-586, 1993.

44. Yachnis A, Berg J, Martinez-Salazar A, Bender BS, Diaz L, Rojiani AM, Eskin TA, Orestein JM. Disseminated microsporidiosis especially infecting the brain, heart, and kidneys: report of a newly recognized pansporoblastic species in two symptomatic AIDS patients. American Journal of Clinical Pathology 106:535-543, 1996. 